Overall in all patients: investigation for cause limited to 3 colonoscopies and 1 ERCP, follow up inadequate in 15 (38\%), mortality 8\%.

Conclusion Management of this serious condition was remarkably poor, with limited use of cultures, inconsistant radiologic intervention, no search for cause, and scanty follow up. Despite this confirmed mortality was just $8 \%$.Guidelines for future management have been drawn up including recommendation that all patients are looked after by the Gastro team.

Disclosure of Interest None Declared

\section{PTU-105 OUTCOME OF CIRRHOTIC PATIENTS ADMITTED TO INTENSIVE CARE UNITS AT HOSPITALS WITHOUT SPECIALIST LIVER SERVICES}

doi:10.1136/gutjnl-2013-304907.195

1."G Beejooa, ${ }^{1} \mathrm{R}$ Oates, ${ }^{2} \mathrm{~L}$ Loo, ${ }^{3} \mathrm{~N}$ Mirza, ${ }^{2} \mathrm{~S}$ Shah, ${ }^{3} \mathrm{C}$ Grimley, ${ }^{4} \mathrm{G}$ Watts, ${ }^{2} \mathrm{~N}$ Prasad, ${ }^{5} \mathrm{~N}$ Kochar, 'A Al Rifai. 'Salford Royal NHS Foundation Trust, Manchester; ${ }^{2}$ Royal Albert Edward Infirmary, Wigan, Manchester; ${ }^{3}$ Royal Blackburn Hospital, Blackburn; ${ }^{4} T$ The University Hospital of South Manchester; ${ }^{5}$ Central Manchester University Hospitals, Manchester, UK

Introduction Patients with liver cirrhosis admitted to an ICU are believed to have a poor prognosis with high mortality despite significant use of resources. Most of the literature to date on this topic has been collected at hospitals with Specialist Liver Units and these results may not be representative of the outcome at general ICUs. A recent prospective study of cirrhotic patients admitted to a tertiary Liver ICU in the UK demonstrated an overall hospital mortality of $59 \%$. The aim of this study was to determine the outcome of cirrhotic patients admitted to non-specialist ICUs.

Methods Data was retrospectively collected from four hospitals in the NW region of the UK without specialist liver ICUs. Patients were identified using the Intensive Care National Audit and Research Unit (ICNARC) database. 61 patients with liver cirrhosis admitted to a general ICU between January 2010 and January 2012 were included in this study.

Results Age range was 30 to 79 years (average 51 years). $80 \%$ of patients were male and alcohol was the commonest aetiology for liver cirrhosis (90\%). The main reason for admission to ICU was for gastrointestinal bleeding (38\%). $51 \%$ of patients had a Child Pugh score of $\mathrm{C}$ on admission to ICU. $46 \%$ of patients had a MELD score between 10 and 19 and $28 \%$ had a score between 20 and $29.79 \%$ of patients required invasive ventilatory support, $49 \%$ required vasopressors and $21 \%$ needed renal replacement therapy. 51\% developed further decompensation of their liver disease during their ICU stay. These included GI bleeding (21\%), hepatic encephalopathy (15\%), HRS (11\%) and SBP (3\%). $67 \%$ of patients had an ICU stay of $<5$ days. A $49 \%$ inpatient mortality rate was observed in our study with sepsis and multi-organ failure being the most common causes of death.

Conclusion Patients with liver cirrhosis admitted to general ICUs have similar rates of mortality compared to those in tertiary liver ICUs. Therefore, admission to such units should not be deemed futile in cirrhotic patients and earlier admission may improve outcome.

Disclosure of Interest None Declared

\section{PTU-106 PATIENT IMPACT OF INFLAMMATION IN PRIMARY BILIARY CIRRHOSIS (PBC): INFLAMIMATORY CYTOKINE LEVELS ARE ELEVATED BUT UNRELATED TO FATIGUE SEVERITY}

doi:10.1136/gutjnl-2013-304907.196

1."G Pells, ' $S$ Ducker, ' $\mathrm{J}$ Palmer, ${ }^{2} \mathrm{~J}$ Newton, ${ }^{1} \mathrm{D}$ Jones on behalf of The UK-PBC Consortium. 'Institute of Cellular Medicine, Newcastle University; ${ }^{2} \mathrm{NIHR}$ Biomedical Research Centre in Ageing \& Chronic Disease, Newcastle-upon-Tyne, UK
Introduction $\mathrm{PBC}$ is characterised by loss of small intrahepatic bile ducts, and in a significant proportion of patients by persistent fatigue. Genes regulating inflammatory pathways have been strongly associated with $\mathrm{PBC}$ in population-scale genetic studies, implicating inflammation in disease pathogenesis. Animal models of cholestasis, a biological process in PBC, have demonstrated fatigue-like behaviour appearing to result from responses to inflammatory cytokine release in the brain. Elevation of inflammatory cytokines has therefore, unsurprisingly, been postulated as an underlying mechanism for fatigue in $\mathrm{PBC}$ as well as other chronic inflammatory conditions. However, more recently data demonstrating that fatigue is not proportional to liver disease severity in PBC has questioned this presumed correlation between inflammation and fatigue. This study aimed to explore the serum cytokine profile in $\mathrm{PBC}$ compared to healthy controls, and to correlate this picture of inflammation status with fatigue severity.

Methods 68 patients from the Newcastle sector of the UK-PBC cohort and 9 healthy controls provided a morning peripheral blood sample and completed the PBC-40, a validated disease-specific quality of life measure with a fatigue domain. Sera were derived using standard protocols and stored at $-80^{\circ} \mathrm{C}$ prior to multiplex cytokine quantification using the MSD platform.

Results $\mathrm{PBC}$ patients showed significant elevation of IFN- $\gamma$ (median 2.4pg/ml[IOR 1.6-15.4] v control 0.7[0.2-1.5], $\mathrm{p}<0.0005)$, IL-6 (1.0pg/ml[0.4-3.3] $\vee 0.5[0-1.5], \mathrm{p}<0.005)$ and TNF- $\alpha(7.1 \mathrm{pg} / \mathrm{ml}[5.5-10.5]$ v $4.3[3.6-5.9], \mathrm{p}<0.001)$. IL-1 $\beta$ was elevated in patients but fell short of significance $(2.3 \mathrm{pg} / \mathrm{ml}$ $[0.2-2.3]$ v $0.5[0.1-0.8], \mathrm{p}=\mathrm{ns})$. Within the PBC group cytokine levels were compared between 21 patients reporting mild fatigue (established using published cut-offs for PBC-40 fatigue domain severity) and 24 patients with severe fatigue. No significant differences were seen between mildly and severely fatigued patients, and for three of the four pro-inflammatory cytokines (IFN$\gamma$,IL-1 $\beta$ and TNF- $\alpha$ ) levels were in fact lower in severely fatigued patients.

Conclusion Serum inflammatory cytokine levels are significantly elevated in $\mathrm{PBC}$, in keeping with inflammation playing a key role in disease pathogenesis. Although the study protocol cannot exclude central nervous system-specific inflammatory mechanisms, no evidence was found to implicate inflammation in the pathogenesis or expression of fatigue in $\mathrm{PBC}$, suggesting a further factor independent of inflammatory disease pathogenesis predisposes certain patients to fatigue.

Disclosure of Interest None Declared.

\section{PTU-107 NAFLD IN PATIENTS WITH SEVERE ASTHMA}

doi:10.1136/gutjinl-2013-304907.197

1,"H Gordon, 'P Patel, ' $\mathrm{J}$ Hull, ' $\mathrm{K}$ Nimako, 'A Menzies-Gow. 'Department of Respiratory Medicine, Royal Brompton Hospital, London, UK

Introduction NAFLD is a spectrum of liver disease that encompasses Nonalcoholic fatty liver (NAFL) and nonalcoholic steatohepatitis (NASH). The pathophysiology is not fully understood, but is believed to be a combination of insulin resistance leading to steatosis and subsequent oxidative injury. Known risk factors include obesity, diabetes and dyslipidaemia.

Severe asthma may entail frequent corticosteroid use and a sedentary lifestyle; both predispose to risk factors implicated in NAFLD. As such we hypothesised a link between asthma and NAFLD, and a possible under detection of NAFLD amongst patients with severe asthma.

Methods We audited the investigation and management of NAFLD amongst patients under the care of the Difficult Asthma Team at the Royal Brompton Hospital. We conducted a retrospective 